\title{
Votation générale FMH: votons OUI!
}

Le projet de loi 04.062 sur le Managed Care est confronté à un problème majeur: personne $n^{\prime}$ en a besoin! - il n'est utile ni aux médecins qui travaillent déjà dans un réseau ni à ceux qui n'en font pas partie. En Suisse, de nombreux médecins (de premier recours) sont déjà regroupés en organisations et réseaux divers. II faut que cette évolution reste à l'avenir libérale et sur une base volontaire.

Sur les 35000 membres que compte la FMH près de 25000 en appellent à la mobilisation du corps médical suisse contre le projet de loi 04.062: quatre associations cantonales (BL, BS, GE, SH), trois sociétés de médecine spécialisée (dermatologues, ophtalmologistes, ORL), ainsi que l'ASMAC et Psica.ch - Il est important de préciser que ces associations et sociétés ne sont pas opposées au principe du Managed Care. Mais elles dénoncent le projet de loi actuel 04.062 et son caractère contraignant.

\section{Pour quelles raisons ces associations et sociétés sont-elles opposées au projet de loi $\mathbf{0 4 . 0 6 2 ?}$}

Parce qu'il abolit le libre choix du médecin, qu'il impose une co-responsabilité budgétaire aux réseaux, qu'il affaiblit l'obligation de contracter et entame le secret médical.

\section{Pourquoi voter OUI à la votation générale:}

- Nous sommes les principaux défenseurs des intérêts de nos patients et nous portons la responsabilité de l'avenir du corps médical.

- Le système existant, permet la coexistence de réseaux de gestion des soins et de réseaux informels, tels qu'on les trouve dans les cabinets de groupe ou les cabinets individuels. Ce modèle a fait ses preuves.

- Le projet de loi 04.062 sur le Managed Care impose une transformation radicale du système de santé helvétique actuel, un système pourtant connu pour la qualité de ses soins médicaux.

- Le projet de loi 04.062 sur le Managed Care abolit le libre choix du médecin et porte préjudice tout autant à nous, les médecins, qu'à nos patients.

- La politique menée jusqu'ici par la FMH, une politique qui s'inspire largement de «la Berne fédérale», ne nous a pas épargné les calamités.

- Des valeurs fondamentales de notre pratique médicale sont mises au rancart parce que la FMH se laisse aller à trop de compromis.

- Tous les sondages et votes réalisés à ce jour auprès des associations cantonales et des sociétés de médecine spécialisée ont abouti à un rejet quasiment unanime d'une obligation du Managed Care.
- Nous n'avons rien à craindre d'un scénario «catastrophe». La FMH peut mener, avec ses 35000 membres, des actions concertées et mobiliser suffisamment de citoyens pour exercer une influence déterminante sur les décisions politiques.

\section{Réflexions corporatistes}

"Celui qui dit A n'est pas obligé de dire B. Il peut aussi reconnaître qu' A était faux.»

(Bertold Brecht)

Il faut reconnaître que les médecins ont euxmêmes proposé au début certains des grands axes du projet de loi 04.062. La thèse FMH «Oui au Managed Care» a été acceptée par la chambre médicale en décembre 2006, à condition toutefois que la co-responsabilité budgétaire reste volontaire.

Lorsqu'un médecin entend travailler de plein gré au sein d'un réseau et assumer une co-responsabilité budgétaire, et que les assurés en sont dûment informés, personne ne trouvera à y redire. Ce type de réseau existe déjà.

Mais aucun médecin ni aucun patient ne doit être contraint d'adhérer à ce système. Et c'est précisément ce que veut imposer le projet de loi 04.062.

\section{II est donc temps de prendre une décision par voie de référendum}

Les deux grands axes du projet de loi (co-responsabilité budgétaire obligatoire dans les réseaux et quotepart différenciée) ne peuvent plus être modifiés par le Parlement.

Nous estimons que ces deux éléments sont inacceptables. Il faut donc envoyer un signal clair au Parlement afin que ce projet soit enterré.

On sait d'ores et déjà que si 04.062 est adopté, un référendum sera lancé: Plusieurs groupes l'ont déjà annoncé.

La co-responsabilité budgétaire obligatoire est une mesure trop radicale pour que nous, médecins, puissions l'accepter! Par conséquent, en cas de référendum, la FMH ne devrait pas rester dans son coin. Elle se doit de mobiliser autant de citoyens que possible contre le projet de loi.

\section{Le projet de loi 04.062 doit être rejeté en bloc}

Avouons-le, ce projet 04.062 est si mal conçu que cela n'a aucun sens de chercher à l'améliorer. Certaines requêtes que le corps médical souhaite encore présenter - comme la demande d'indépendance face aux réseaux (Motion Cassis) - sont certes méritoires mais elles ne suppriment en rien les éléments inacceptables du 04.062. 


\section{On ferait mieux de défendre nos valeurs fondamentales au lieu de se perdre dans des arrières-pensées tactiques}

Il y a trois ans de cela, la FMH s'était battue avec succès pour le maintien du libre choix du médecin (vota-

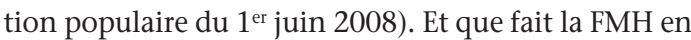
2011? Elle se déclare favorable à un projet de loi qui menace le libre choix du médecin!

On veut nous faire croire qu'en utilisant des manigances «tactiques» on va pouvoir vouloir empêcher la suppression de l'obligation de contracter. Ce scénario «catastrophe» est cousu de fil blanc: On prétend être obligé de choisir entre deux maux. Non. C'est maintenant que la FMH a l'occasion de montrer sa force, en affichant une unité qui s'appuie légitimement sur sa base.

Laissons la «tactique» aux hommes politiques. En tant que médecins (la FMH compte 35000 membres), dans une Suisse démocratique, nous pouvons influer sur les décisions politiques en défendant activement nos objectifs principaux. A nous d'éviter la voie glissante des compromis trop faciles. Nous ne pouvons négocier certaines valeurs fondamentales telles que le maintien d'une relation de confiance entre le médecin et son patient.

\section{Le projet de loi 04.062 est inutile. II a pour seul but de rationner les prestations de la LAMal}

Le projet de loi 04.062 n'est pas la solution qu'il faut pour faire face à l'augmentation des dépenses de santé, qui est due d'abord à l'évolution démographique.

Les médecins ont déjà leurs réseaux. Et ils sont conscients des coûts, étant tenus d'appliquer les critères $\mathrm{EAE}$, que ce soit au sein de l'un des réseaux structurés ou dans un réseau informel. Le projet de loi 04.062 est donc inutile! La quote-part différenciée devrait pousser les patients et les médecins à s'affilier à un réseau officiel. Mais les assureurs ne vont pas manquer de serrer la vis dans les réseaux, par le biais de la co-responsabilité obligatoire. Les objectifs budgétaires

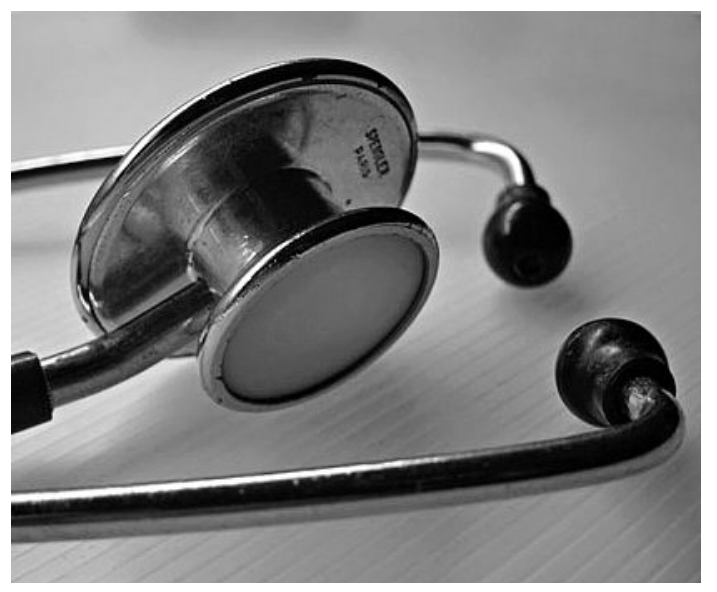

se durciront au fil du temps et les médecins seront soumis à une pression inexorable. Ce qui se traduira par un rationnement des soins. Il sera ainsi facile pour les politiciens et l'opinion publique de se décharger, sur le dos des médecins, des décisions ingrates et difficiles portant sur le type de rationnement à effectuer. On considère en général que la responsabilité médicale et la responsabilité économique (médecin traitant contre médecin-conseil) doivent restées séparées. Si cette distinction disparaît et que les décisions médicales sont largement dictées par des mobiles économiques, alors la relation de confiance entre le médecin et son patient s'en trouvera ébranlée.

\section{Pour un corps médical uni}

Le projet de loi 04.062, monte les médecins les uns contre les autres. On prétend que la qualité des soins de santé serait meilleure au sein des réseaux officiels qu'en dehors. C'est là une supposition qui n'a encore jamais été prouvée, pas plus que l'affirmation selon laquelle les réseaux de soins intégrés seraient plus rentables.

Neuf sociétés médicales comptant près de 25000 membres demandent que la FMH s'engage systématiquement contre le projet de loi 04.062 sur le Managed Care tel que le prévoit le Parlement, contre l'abolition du libre choix du médecin que cela implique (par le biais de la quote-part différenciée), contre la co-responsabilité obligatoire dans les réseaux, contre la suppression de l'obligation de contracter dans les réseaux et contre l'érosion du secret médical. La FMH doit s'engager activement et ouvertement en faveur de ces objectifs, ce qui implique également le soutien à un éventuel référendum contre ce projet de loi 04.062.

Ärztegesellschaft Baselland, Medizinische Gesellschaft Basel, Association des Médecins du Canton de Genève, Kantonale Ärztegesellschaft Schaffhausen, Société Suisse de Dermatologie, Société Suisse d'Ophtalmologie, Société Suisse d'ORL et Psica.ch

\section{OUI à ma profession:}

\section{- Ma liberté \\ - Mon indépendance \\ - Mon avenir}

OUI à mes patients:

- $\quad$ Leur liberté

- $\quad$ Leur santé

- $\quad$ Leur choix

\section{C'est à nous de décider - Votons OUI!}




\section{Oui à la demande de votation générale}

L'ASMAC salue la prise en charge intégrée. Pour les malades chroniques, il est essentiel que des réseaux de soins soient mis en place au sein desquels les différents fournisseurs de prestations (médecins de premier recours, soins à domicile, cabinets spécialisés, cliniques, etc.) collaborent de façon optimale. Cela permet d'améliorer la qualité des soins et d'éviter des doublons coûteux.

Pour I'ASMAC cependant, le projet Managed Care va dans la mauvaise direction.

\section{Pourquoi maintenant?}

Avec leur menace de référendum, les membres de la FMH envoient un signal clair aux Conseillers/-ères nationaux et des Etats en prévision du vote final au parlement le 30 septembre. Il est temps que les médecins manifestent leur opposition au projet Managed Care mal conçu.

Selon l'ASMAC, les arguments suivants sont essentiels à cet égard:

\section{La suppression du libre choix du médecin}

Le parlement prévoit actuellement pour 2012 de punir financièrement les patients qui ne veulent pas s'assurer dans un modèle de prise en charge intégrée, notamment par le biais de quotes-parts «différenciées». Comme il est prévu de faire payer une quotepart deux à trois fois plus élevée à tous les assurés qui ne s'affilient pas à un réseau, le libre choix du médecin devrait donc être payé au prix fort à l'avenir. C'est-à-dire que beaucoup de patients seraient pratiquement obligés de s'affilier à un modèle Managed Care.

\section{Pour I'ASMAC, le projet Managed Care va dans la mauvaise direction.}

\section{Le problème de la coresponsabilité budgé- taire}

Les réseaux de soins intégrés doivent obligatoirement supporter une coresponsabilité budgétaire. Ce qui commence comme une coresponsabilité se transformera vite en pleine responsabilité dans le cadre des efforts d'économie.
L'ASMAC met en garde contre la responsabilité budgétaire pour les raisons suivantes:

- elle conduit à un rationnement caché et à une perte inévitable de la qualité;

- il en résulte une incitation au sous-approvisionnement;

- les fournisseurs de prestations sont poussés à pratiquer une sélection des risques;

- la relation de confiance entre médecin et patient est troublée, car les responsabilités médicales et économiques ne sont plus dissociées.

\section{Le problème de la liberté de contracter}

Selon le parlement, les caisses-maladie pourront décider arbitrairement avec quels réseaux de soins (avantageux) elles souhaitent conclure des conventions et avec lesquels pas. L'obligation de contracter serait donc vidée de sa substance.

Il est temps que les médecins manifestent leur opposition au projet Managed Care mal conçu.

\section{Le problème du secret médical}

En outre l'échange des données entre les réseaux qui est exigé pour «optimiser» les traitements, aussi connu comme «patient transparent», affaiblit considérablement le secret médical, c'est-à-dire la relation de confiance entre médecin et patient. En particulier pour le traitement des patients stigmatisés, par ex. atteints de maladies psychiques, le transfert de données déjà pratiqué en ce moment dans les réseaux peut avoir des répercussions fâcheuses sur la relation entre médecin et patient.

\section{Conclusion}

Ces projets de politique de la santé mettent en péril notre approvisionnement en soins, qui est excellent en comparaison mondiale. Le parlement ne doit pas décider à lui seul de la suppression du libre choix du médecin qui a fait ses preuves en Suisse. Le peuple doit impérativement être consulté avant un changement aussi profond et irréversible du système de santé suisse. 